\title{
Application of the out-of-phase absorption mode to separating overlapping EPR signals with different $T_{1}$ values
}

\author{
V.A. Livshits ${ }^{\mathrm{b}}$, D. Marsh ${ }^{\mathrm{a}, *}$ \\ ${ }^{a}$ Max-Planck-Institut für biophysikalische Chemie, Abt. Spektroskopie, 37077 Göttingen, Germany \\ ${ }^{\mathrm{b}}$ Centre of Photochemistry, Russian Academy of Sciences, 117427, Moscow, Russian Federation
}

Received 21 December 2004; revised 27 April 2005

Available online 8 June 2005

\begin{abstract}
The use of $90^{\circ}$-out-of-phase first-harmonic absorption $\left(V_{1}^{\prime}-\right)$ EPR to resolve the spectra from nitroxide spin labels with differing $T_{1}$-relaxation times is described. Non-linear $V_{1}^{\prime}$-EPR spectra recorded under moderate saturation have sharper lines compared with the in-phase $V_{1}$-EPR spectra, and amplitudes that preferentially enhance components with longer $T_{1}$-relaxation. Discrimination between $V_{1}^{\prime}$-spectral components can be increased further by means of selective paramagnetic relaxation enhancement agents. Examples are given of biophysical applications to double labelling in single-component membranes and phase separation in two-component membranes, to lipid-peptide complexes, and to binding of spin-labelled reagents. It is concluded that optimal resolution in $V_{1}^{\prime}$-EPR spectroscopy is obtained at relatively low Zeeman modulation frequencies $(20-30 \mathrm{kHz})$ and moderate saturation $\left(H_{1} \sim 0.2-0.3 \mathrm{G}\right)$.
\end{abstract}

(C) 2005 Elsevier Inc. All rights reserved.

Keywords: Non-linear EPR; First-harmonic 90-out-of-phase; Spin label; Lateral phase separation; Lipid-peptide interaction

\section{Introduction}

One of the important problems in spin-label EPR spectroscopy of microheterogeneous systems, in particular of lipid membranes, concerns the presence of two (or more) overlapping EPR signals that correspond either to different paramagnetic species or to the localization of the same spin label in different environments. Examples frequently encountered are: paramagnetic ions that have strong background EPR signals at fields close to that for nitroxide spin labels, overlapping EPR spectra of a spin-labelled lipid or of two different lipid spin labels, partition of a spin label between different environments (e.g., aqueous phase and membrane, lipid bilayer and the boundary layer at the protein/lipid interface, or different lipid phases in two- or three-component lipid

\footnotetext{
* Corresponding author. Fax: +495512011501

E-mail address: dmarsh@gwdg.de (D. Marsh).
}

mixtures), and covalently bound spin labels in different conformational states or binding sites.

Conventionally, analysis of such systems has been performed by difference spectroscopy in the linear EPR regime. For its success, this requires a reasonable degree of resolution of the component spectra and knowledge of the lineshape of at least one of the components. This is, however, not always the case. We suggest that non-linear out-of-phase first-harmonic absorption EPR signals $\left(V_{1}^{\prime}\right)$ may be used to solve this problem, in several cases, and thus to increase the range of multi-component systems that can be analyzed. Earlier, first-harmonic EPR signals detected in phase-quadrature with the Zeeman modulation, under conditions of partial microwave saturation, were used to determine spin-lattice relaxation times [1]. Sensitivity of the $V_{1}^{\prime}$-EPR spectra also to molecular motion was investigated by spectral simulations for nitroxide spin labels, over the entire range of rotational correlation times 
[2]. It was shown that determination of the effective spin-lattice relaxation times from $V_{1}^{\prime}$-EPR spectra is less dependent on rotational mobility than for other non-linear CW-EPR methods (see also [3]), especially at lower Zeeman modulation frequencies. Experimental measurements on spin labels in the slow, intermediate, and fast motional regimes of molecular rotation were used to test and validate the method. Measurement of the spin-lattice relaxation enhancements of bound spin-labelled fatty acids by using $V_{1}^{\prime}$-EPR spectroscopy allowed us to obtain information on the microtopography of the fatty acid binding sites in human serum albumin [4]. The $V_{1}^{\prime}$-EPR approach was further generalized to anisotropic rotation of spin labels [5], and the validity of the method was tested by using oxygen-induced relaxation enhancements of spin labels in membranes that were measured simultaneously by this and the progressive $\mathrm{CW}$-saturation method [6].

As regards the problem of spectral separation of overlapping EPR signals, the potential of the $V_{1}^{\prime}$-approach is based on two properties of the $V_{1}^{\prime}$-EPR signals that will be deduced below: (i) spectral resolution in the $V_{1}^{\prime}$-spectra is higher than for the corresponding in-phase signals, and (ii) there is a rather strong dependence of the intensity of the $V_{1}^{\prime}$-spectrum on spin-lattice relaxation time [7]. The intensity of one of two overlapping signals will be relatively much higher in the $V_{1}^{\prime}$-EPR spectrum than in the conventional $V_{1}$-EPR spectrum, if $T_{1}$ of one signal is significantly longer than for the other.

The aim of this paper is to develop the first-harmonic, out-of-phase $V_{1}^{\prime}$-approach to resolution enhancement in detail and to investigate its robustness for several spinlabelled systems of biological relevance. In particular, we investigate lateral phase separation in mixed lipid membranes composed of dimyristoyl phosphatidylcholine (DMPC) and distearoyl phosphatidylcholine (DSPC), and lipid-peptide interactions in mixtures of gramicidin A and DMPC. For the case of lateral phase separation, the non-linear relaxation-enhanced spectra reveal a spin-labelled lipid component that is masked in the conventional EPR spectra. For the GA/DMPC system, heterogeneity on the $T_{1}$-timescale is revealed in the lipid-peptide interactions.

\section{Theoretical background}

We consider here the reasons why first-harmonic outof phase EPR signals can be useful for separation of overlapping EPR spectra. As in [1] we confine ourselves, for simplicity, to a single-line EPR spectrum in the absence of molecular motion. Expressions for the absorption lineshapes detected at the first-harmonic, both in-phase $\left(V_{1,1}\right)$ and out-of-phase $\left(V_{1,1}^{\prime}\right)$ with respect to the field modulation, and under conditions of partial microwave power saturation, can be obtained by iterative solution of the Bloch equations (see $[8,9]$ ):

$$
\begin{aligned}
V_{1,1}(H)= & -V_{0,0}\left(\gamma_{e} H-\omega\right) T_{2} \\
& \times \frac{2\left(\gamma_{e} H-\omega\right)^{2} T_{2}^{2}+2 A-B \omega_{m}^{2} T_{2}^{2}}{\left[\left(\gamma_{e} H-\omega\right)^{2} T_{2}^{2}+A\right]^{2}+B^{2} \omega_{m}^{2} T_{2}^{2}}, \\
V_{1,1}^{\prime}(H)= & V_{0,0}\left(\gamma_{e} H-\omega\right) \omega_{m} T_{2}^{2} \\
& \times \frac{\left(\gamma_{e} H-\omega\right)^{2} T_{2}^{2}+A+2 B}{\left[\left(\gamma_{e} H-\omega\right)^{2} T_{2}^{2}+A\right]^{2}+B^{2} \omega_{m}^{2} T_{2}^{2}}
\end{aligned}
$$

with

$A=1-\omega_{m}^{2} T_{2}^{2}+\gamma_{e}^{2} H_{1}^{2} T_{1} T_{2} \frac{1+\omega_{m}^{2} T_{1} T_{2}}{1+\omega_{m}^{2} T_{1}^{2}}$

and

$B=-2+\gamma_{e}^{2} H_{1}^{2} T_{1} \frac{T_{1}-T_{2}}{1+\omega_{m}^{2} T_{1}^{2}}$,

where $T_{1}$ and $T_{2}$ are the longitudinal and transverse relaxation times, respectively, $\omega$ and $\omega_{m}$ are the angular microwave and field modulation frequencies, respectively, and $H_{1}$ and $H$ are the microwave and static magnetic field strengths, respectively. In Eqs. (1) and (2), the zeroth harmonic solution, $V_{0,0}$, is given by the normal slowpassage absorption lineshape, i.e.,

$V_{0,0}(H)=\frac{\gamma_{e} H_{1} T_{2}}{1+\left(\gamma_{e} H-\omega\right)^{2} T_{2}^{2}+\gamma_{e}^{2} H_{1}^{2} T_{1} T_{2}}$,

where the slow-passage saturation parameter in the denominator is $s=\gamma_{e}^{2} H_{1}^{2} T_{1} T_{2}$.

We can simplify the analytical expressions for $V_{1,1}$ and $V_{1,1}^{\prime}$ by using the reasonable approximation that $A^{2} \gg B^{2} \omega_{m}^{2} T_{2}^{2}$, which always holds for $\omega_{m} T_{1}<1$ (and also for $\omega_{m} T_{1}>1$, in the case of low saturation, i.e., $s \leqslant 1$ ) [1]. Additionally, we use the relations:

$T_{2} / T_{1} \ll 1, \omega_{m} T_{2} \ll 1$ and $\omega_{m}^{2} T_{1} T_{2} \ll 1$,

which hold for nitroxides in liquid solutions and in membranes, where the usual range of relaxation times is $5 \times 10^{-7}-10^{-5} \mathrm{~s}$ for $T_{1}$ and $10^{-8}-10^{-7} \mathrm{~s}$ for $T_{2}[10$ 13]. These inequalities lead to approximations for $A$ and $B$ that are given by:

$A \approx 1+\frac{\gamma_{e}^{2} H_{1}^{2} T_{1} T_{2}}{1+\omega_{m}^{2} T_{1}^{2}}$

$B \approx-2+\frac{\gamma_{e}^{2} H_{1}^{2} T_{1}^{2}}{1+\omega_{m}^{2} T_{1}^{2}}$.

We consider the case of low modulation frequency, $\omega_{m} /$ $2 \pi \leqslant 30 \mathrm{kHz}$, which was concluded earlier to be optimal for spin-lattice relaxation measurements in the $V_{1,1}^{\prime}$-mode [1,2]. For these frequencies, the condition: $\omega_{m} T_{1}<1, \omega_{m}^{2} T_{1}^{2} \ll 1$, holds for $T_{1}<5 \mu \mathrm{s}$. 
In this case:

$A \approx 1+\gamma_{e}^{2} H_{1}^{2} T_{1} T_{2}$

$B \approx-2+\gamma_{e}^{2} H_{1}^{2} T_{1}^{2}$,

and the expressions for $V_{1,1}$ and $V_{1,1}^{\prime}$ become

$$
V_{1,1}(H) \cong-2 \cdot \frac{\gamma_{e} H_{1}\left(\gamma_{e} H-\omega\right) T_{2}^{2}}{\left[1+\left(\gamma_{e} H-\omega\right)^{2} T_{2}^{2}+\gamma_{e}^{2} H_{1}^{2} T_{1} T_{2}\right]^{2}}
$$

and

$$
\begin{aligned}
& V_{1,1}^{\prime}(H)=\gamma_{e} H_{1}\left(\gamma_{e} H-\omega\right) \omega_{m} T_{2}^{3} \\
& \quad \times\left(\frac{\gamma_{e}^{2} H_{1}^{2} T_{1}\left(2 T_{1}+T_{2}\right)+\left(\gamma_{e} H-\omega\right)^{2} T_{2}^{2}-3}{\left[1+\left(\gamma_{e} H-\omega\right)^{2} T_{2}^{2}+\gamma_{e}^{2} H_{1}^{2} T_{1} T_{2}\right]^{3}}\right),
\end{aligned}
$$

respectively. Representative in-phase and out-of-phase lineshapes predicted by these two equations are given in Fig. 1. It is seen immediately that the out-of-phase $\left(V_{1,1}^{\prime}\right)$ EPR spectrum is considerably narrower than the in-phase $\left(V_{1,1}\right)$ EPR spectrum, especially in the outer wings. This line narrowing forms a basis, quite separate from $T_{1}$-discrimination, for improved resolution in firstharmonic out-of-phase EPR spectroscopy.

We note that for $T_{1} \cong 10^{-6} \mathrm{~s}$, which is typical for nitroxide spin labels, the inequality $2 \gamma_{e}^{2} H_{1}^{2} T_{1}^{2} \gg 3$ holds for microwave field strengths $H_{1}>0.07 \mathrm{G}$. The latter value is well below the optimal $H_{1}$ normally used for $V_{1,1}^{\prime}$ measurements, which routinely is in the range $0.3-0.5 \mathrm{G}$ $[1,2]$. Therefore, the dominant term in the numerator on the right of Eq. (12) is $2 \gamma_{e}^{2} H_{1}^{2} T_{1}^{2}$, for field scans up to $\left(\gamma_{e} H-\omega\right) T_{2} \leqslant 5-10$. The peak-to-peak linewidths of the $V_{1,1}$-and $V_{1,1}^{\prime}$-components determined from Eqs. (11) and (12) are hence given by the following expressions, respectively:

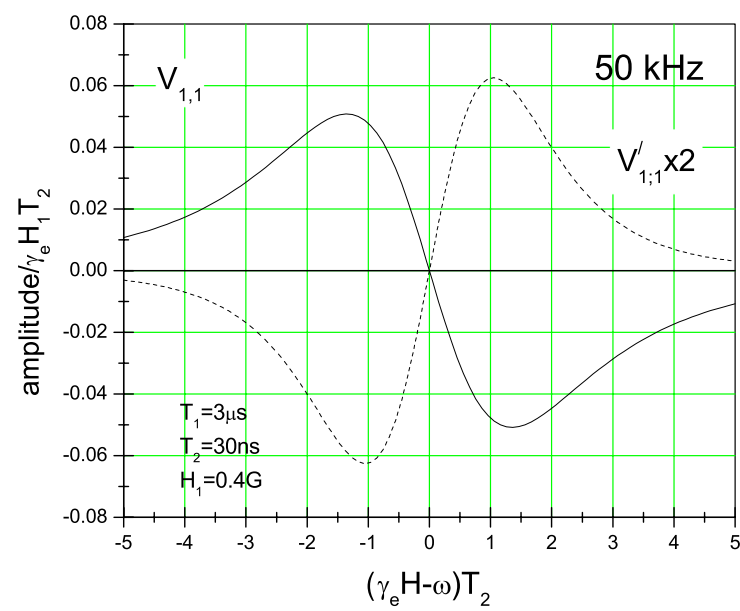

Fig. 1. First harmonic in-phase $\left(V_{1,1}\right)$ and out-of-phase $\left(V_{1,1}^{\prime}\right)$ spectral lineshapes calculated according to Eqs. (11) and (12), respectively. Zeeman modulation frequency is $\omega_{m} / 2 \pi=50 \mathrm{kHz}$, and microwave magnetic field $H_{1}=0.4 \mathrm{G}$. Relaxation times are $T_{1}=3 \times 10^{-6} \mathrm{~s}$ and $T_{2}=3 \times 10^{-8} \mathrm{~s}$. In subsequent displays of experimental spectra, the phase of the $V_{1}^{\prime}$-spectrum is reversed and the amplitude is normalized to that of the $V_{1}$-spectrum.

$$
\begin{aligned}
& \Delta H_{\mathrm{pp}}=\frac{2}{\sqrt{3}} \frac{\sqrt{1+\gamma_{e}^{2} H_{1}^{2} T_{1} T_{2}}}{\gamma_{e} T_{2}}, \\
& \Delta H_{\mathrm{pp}}^{\prime}=\frac{2}{\sqrt{5}} \frac{\sqrt{1+\gamma_{e}^{2} H_{1}^{2} T_{1} T_{2}}}{\gamma_{e} T_{2}} .
\end{aligned}
$$

It is seen that the peak-to-peak linewidth of the out-ofphase $V_{1,1}^{\prime}$-component is less than that of the in-phase $V_{1,1}$-component. Moreover, for relatively low degrees of microwave saturation, $s=\gamma_{e}^{2} H_{1}^{2} T_{1} T_{2}<2 / 3$, the outof-phase linewidth is smaller than that measured by conventional EPR at low power, viz., $\Delta H_{\mathrm{pp}}^{0}=2 /\left(\sqrt{3} \gamma_{e} T_{2}\right)$. It can also be seen from Eqs. (11) and (12) that the wings of the out-of-phase $V_{1,1}^{\prime}$-spectrum decay more rapidly than do those of the in-phase $V_{1,1}$-spectrum. For field scans in the range 5-10 $\geqslant\left(\gamma_{e} H-\omega\right) T_{2} \gg$ $\sqrt{1+\gamma_{e}^{2} H_{1}^{2} T_{1} T_{2}}$, the leading term in Eq. (12) (with $\left.2 \gamma_{e}^{2} H_{1}^{2} T_{1}^{2} \gg 3, s\right)$ gives a lineshape $V_{1,1}^{\prime} \sim 1 /\left(\gamma_{e} H-\omega\right)^{5}$ for the out-of-phase signal, as compared with $V_{1,1} \sim 1$ / $\left(\gamma_{e} H-\omega\right)^{3}$ deduced from Eq. (11) for the in-phase lineshape. In the case of spectral overlap, both the above factors should result in better resolution of the out-of-phase $V_{1,1}^{\prime}$-spectra as compared with the in-phase $V_{1,1}$-spectra (see Fig. 1).

The dependences on $T_{1}$ and $H_{1}$ of the peak-to-peak amplitudes in the $V_{1,1^{-}}$and $V_{1,1}^{\prime}$-spectra are given, from Eqs. (11)-(14), by:

$V_{1,1}^{\mathrm{pp}} \cong \frac{9}{4 \sqrt{3}} \frac{\gamma_{e} H_{1} T_{2}}{\left(1+\gamma_{e}^{2} H_{1}^{2} T_{1} T_{2}\right)^{3 / 2}}$

and

$V_{1,1}^{\prime \mathrm{pp}} \cong \frac{25 \sqrt{5}}{54} \omega_{m} \frac{\gamma_{e}^{3} H_{1}^{3} T_{1}^{2} T_{2}^{2}}{\left(1+\gamma_{e}^{2} H_{1}^{2} T_{1} T_{2}\right)^{5 / 2}}$,

respectively. It follows from Eqs. (14) and (16) that, in order to increase the spectral resolution in the $V_{1,1}^{\prime}$-mode for two overlapping EPR signals with different $T_{1}$ values, one must minimize the line broadening arising from microwave saturation. Moreover, at moderate degrees of saturation $s=\gamma_{e}^{2} H_{1}^{2} T_{1} T_{2}<1$, in contrast to the $V_{1,1}$-signal, the $V_{1,1}^{\prime}$-amplitude depends strongly on $T_{1}$ which is confirmed by exact numerical calculation. Then the intensity of a signal in the outof-phase $V_{1,1}^{\prime}$-spectrum will be relatively much higher than that in the in-phase $V_{1,1}$-spectrum, if $T_{1}$ for this signal is significantly longer than for the other signals.

From Eqs. (15) and (16), the out-of-phase to in-phase ratio of the first-harmonic peak-to-peak amplitudes is given by

$\rho_{1}^{\prime}\left(\Delta H^{\mathrm{pp}}\right)=\frac{V_{1,1}^{\mathrm{pp}}}{V_{1,1}^{\mathrm{pp}}} \cong 0.8 \times \frac{\omega_{m} T_{1}}{1+1 /\left(\gamma_{e}^{2} H_{1}^{2} T_{1} T_{2}\right)}$

for low modulation frequencies. This relation demonstrates rather directly the selective enhancement of 
components with longer $T_{1}$ in the out-of-phase $V_{1}^{\prime}$-EPR spectrum, relative to the in-phase $V_{1}$-EPR spectrum recorded at the same microwave power. It also shows that, quite generally, the relative intensity of the outof-phase spectrum depends rather strongly not only on $T_{1}$ but also on the degree of saturation, $s=\gamma_{e}^{2} H_{1}^{2} T_{1} T_{2}$, and the modulation frequency, $\omega_{m}$. For moderately low saturation $\left(H_{1}=0.3 \mathrm{G}\right)$, and relatively short relaxation times $\left(T_{1}=1 \mu \mathrm{s}, T_{2}=10 \mathrm{~ns}\right)$, Eq. (17) predicts $V_{1,1}^{\mathrm{pp}} / V_{1,1}^{\mathrm{pp}} \cong 0.03$ at $30 \mathrm{kHz}$ modulation frequency; whereas for the conditions of Fig. 1 the out-of-phase/in-phase ratio is almost 20 times larger than this. At a modulation frequency of $100 \mathrm{kHz}$ and moderately strong saturation, the out-of-phase and in-phase signals can become of comparable intensity. Even under conditions giving rise to rather low out-of-phase/in-phase ratios in the non-linear regime, useable signal strength can be obtained in the out-ofphase $V_{1}^{\prime}$-EPR spectrum because the latter is routinely recorded at a much higher microwave power than are conventional spin-label EPR spectra [1,2]. For the example given above, it is predicted from Eqs. (15) and (16) that the amplitude of the out-of-phase $V_{1}^{\prime}$-spectrum is $34 \%$ that of the conventional in-phase $V_{1}$-spectrum recorded at a normal subsaturating microwave power corresponding to $H_{1}=0.02 \mathrm{G}$. This is 10 times greater than the out-of-phase/in-phase $\rho_{1}^{\prime}$-ratio predicted for the non-linear first-harmonic spectra recorded at $H_{1}=0.3 \mathrm{G}$.

The preceding relations were obtained under the conditions given by Eqs. (9) and (10), for low modulation frequencies. If the inequalities $\omega_{m} T_{1}<1, \omega_{m}^{2} T_{1}^{2} \ll 1$ do not hold, then $B$ is given by Eq. (8), and the dependence of the $V_{1,1}^{\prime}$-amplitude on $T_{1}$ in Eq. (2) becomes weaker than that predicted by the approximation given in Eq. (12). This result explains the experimental findings (see below) that better signal separation is achieved at low modulation frequencies. However, the strength of the out-of-phase $V_{1,1}^{\prime}$-signal increases with increasing modulation frequency (see Eqs. (2) and (12)). As a compromise between efficiency of signal separation and concentration sensitivity, modulation frequencies of $20-30 \mathrm{kHz}$ are optimal.

The above considerations apply to homogeneously broadened lines. Inhomogeneous broadening of the spectral lines produces two effects. The first one is trivial: a decrease in spectral resolution because of broadening. The second is that inhomogeneous broadening decreases the power dependence on $T_{1}$ and $T_{2}$ relative to that given by the denominators of the homogeneously broadened $V_{1,1^{-}}$and $V_{1,1}^{\prime}$-signals in Eqs. (11) and (12) (see [14]), and therefore the $T_{1}$ dependence of the $V_{1,1}^{\prime}$-signal increases in the presence of inhomogeneous broadening because of the first term in the numerator of Eq. (12). This result is confirmed by exact numerical calculations.

\section{Materials and methods}

The spin label TEMPOstearate (4-octadecanoyl2,2,6,6-tetramethylpiperidine- $N$-oxyl) was obtained from Molecular Probes (Eugene, OR). Spin-labelled phosphatidylcholines, $n$-PCSL (1-acyl-2-[n-(4,4-dimethyloxazolidine- $N$-oxyl)stearoyl]-sn-glycero-3-phosphocholine), were synthesized according to [15]. Synthetic phosphatidylcholines, 1,2-dimyristoyl-sn-glycero-3-phosphocholine (DMPC), and 1,2-distearoyl-sn-glycero-3-phosphocholine (DSPC) were from Avanti Polar Lipids (Alabaster, AL). Gramicidin A (GA), spin-labelled maleimide (3-maleimido-proxyl), human serum albumin, and $\mathrm{Ni}\left(\mathrm{ClO}_{4}\right)_{2}$ were from Sigma Chemical (St. Louis, MO). Chromium oxalate was synthesized according to [16].

TEMPOstearate (TS) or spin-labelled phosphatidylcholines, $n$-PCSL ( $n=6,14$ or 16$)$ were incorporated in bilayer membranes of DMPC, DMPC/DSPC or GA/DMPC at a relative concentration of $0.5 \mathrm{~mol} \%$, (see e.g., [15]). All membrane dispersions were saturated with argon. Aliquots of the samples were loaded into $50 \mu \mathrm{l}, 0.7 \mathrm{~mm}$ i.d., glass capillaries and flushed with argon. Sample sizes were trimmed to $5 \mathrm{~mm}$ length to avoid inhomogeneities in the $H_{1}$ and $H_{m}$ fields [17].

EPR spectra were recorded at a microwave frequency of $9 \mathrm{GHz}$ on a Bruker EMX EPR spectrometer (Bruker Biospin, Karlsruhe) equipped with nitrogen gas flow temperature regulation. Sample capillaries were positioned along the symmetry axis of the standard $4 \mathrm{~mm}$ quartz EPR sample tube that contained light silicone oil for thermal stability. Temperature was measured with a fine-wire thermocouple located within the capillary in contact with the sample. Samples were centered in the $\mathrm{TE}_{102}$ rectangular microwave cavity and all spectra were recorded under critical coupling conditions. The root-mean-square microwave magnetic field $\left\langle H_{1}^{2}\right\rangle^{1 / 2}$ at the sample was measured as described in [17], and corrections were made for the cavity $Q$ as described in the same reference. The in-phase and out-ofphase EPR spectra were recorded in the first-harmonic absorption mode at modulation frequencies of 100,30 , and $20 \mathrm{kHz}$. The modulation field measured at the sample was $0.32 \mathrm{G} \mathrm{p}-\mathrm{p}$ [17]. The procedures required for correct recording of the $V_{1}^{\prime}$-signals, especially at low $H_{1}$-values, were described in [1].

\section{Results and discussion}

Several lipid or lipid/protein experimental systems were studied that demonstrate an increase in spectral resolution, and/or preferential enhancement of a particular component, when using the non-linear first-harmonic, out-of-phase $V_{1}^{\prime}$-display for these heterogeneous systems.

First, however, we present the results of spectral simulations that provide calibrations of the dependence of 
the first-harmonic out-of-phase to in-phase ratios on $T_{1}$ and rotational mobility. These apply to experimental conditions that are likely to optimize resolution in the $V_{1}^{\prime}$-EPR spectra (see Section 2). Previous calibrations apply solely to high $H_{1}$-values that are designed to optimize $T_{1}$-determinations from single-component spectra $[1,2]$.

\subsection{Calibration of out-of-phaselin-phase amplitude ratios}

The effects of rotational motion on the $T_{1}$-dependence of the first-harmonic out-of-phase to in-phase amplitude ratios, $\rho_{1}^{\prime}\left(M_{I}\right)$, were investigated previously by spectral simulation for large $H_{1}$ amplitudes [2]. By using the same uncorrelated jump model, we have now performed similar simulations for lower values of $H_{1}$ and the intermediate modulation frequencies that favor improved resolution in multi-component $V_{1}^{\prime}$-EPR spectra. The $T_{1}$-dependences of the low-field amplitude ratio are presented in Fig. 2, for a modulation frequency of $20 \mathrm{kHz}, H_{1}=0.18-0.36 \mathrm{G}$, and different values of the rotational frequency $f_{\mathrm{R}}$. Just as found previously at

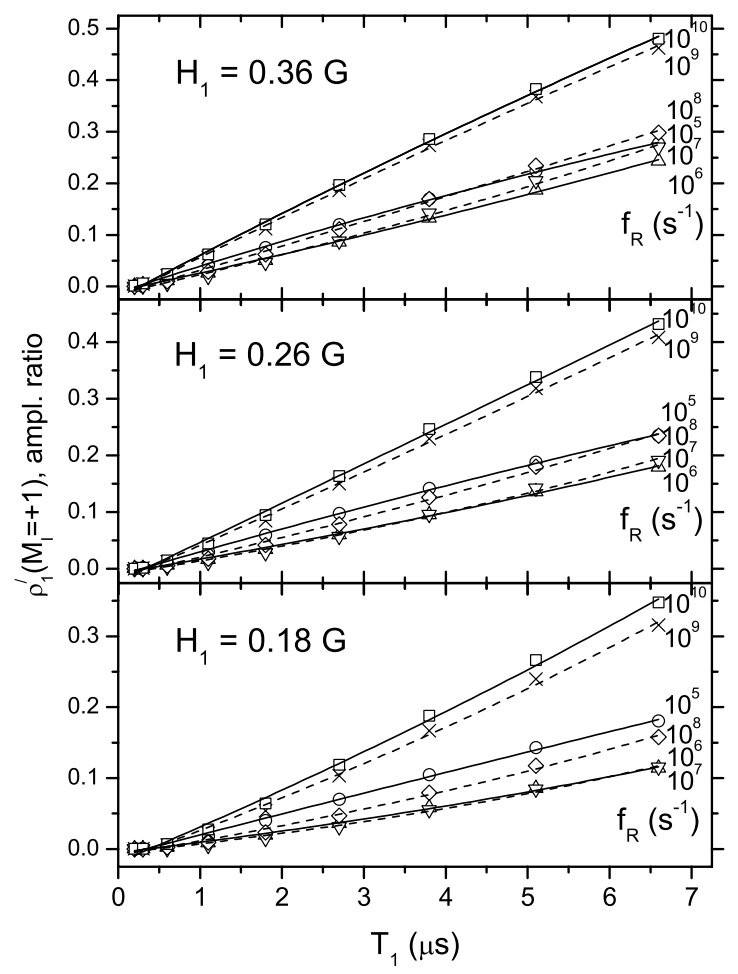

Fig. 2. Simulated dependences on $T_{1}$ of the ratio, $\rho_{1}^{\prime}\left(M_{I}\right)$, of the amplitudes of the low-field $\left(M_{I}=+1\right)$ hyperfine component of the outof-phase and in-phase first-harmonic EPR spectra, for different values of the rotational frequency $f_{\mathrm{R}}$ as indicated. Upper to lower panels: $H_{1}=0.36,0.26$, and $0.18 \mathrm{G}$. Zeeman modulation frequency is $20 \mathrm{kHz}$; homogeneous linewidth: $\Delta H_{0}\left(=1 / \gamma_{e} T_{2,0}\right)=1.0 \mathrm{G}$, with an inhomogeneous broadening of $1.4 \mathrm{G}$ peak-to-peak. Solid and dashed lines are non-linear least-squares fits of Eq. (18) for $m=1$, with the fitting parameters given in Table 1. higher $H_{1}$-fields, the $\rho_{1}^{\prime}$ ratio has a non-monotonic dependence on rotational frequency. The $M_{I}=+1$ amplitude ratio decreases with decreasing $f_{\mathrm{R}}$ over the range $10^{10}-10^{7} \mathrm{~s}^{-1}$, but then increases on decreasing $f_{\mathrm{R}}$, beyond $f_{\mathrm{R}} \leqslant 10^{7} \mathrm{~s}^{-1}$. The minimum, corresponding to maximum spectral diffusion, lies in the region of $f_{\mathrm{R}} \approx 10^{7} \mathrm{~s}^{-1}$.

Calibrations for the $T_{1}$-dependence of the $\rho_{1}^{\prime}$-ratio that are valid for all rotational frequencies, $f_{\mathrm{R}}$, were introduced previously by using the following expression $[1,2]$

$\rho_{1}^{\prime}\left(M_{I}\right)=\rho_{1}^{o \prime}\left(M_{I}\right)+\frac{a_{1}^{\prime}\left(M_{I}\right) T_{1}^{m}}{1+b_{1}^{\prime}\left(M_{I}\right) T_{1}^{m}}$,

where the exponent, $m$, depends on the modulation frequency. With a high microwave amplitude, $H_{1}=0.5 \mathrm{G}$, it was found that: $m=1.6$ for $\omega_{m} / 2 \pi=100 \mathrm{kHz}$ and $m=1.3$ for $\omega_{m} / 2 \pi=25 \mathrm{kHz}$ [2]. For lower microwave amplitudes, $H_{1} \leqslant 0.36 \mathrm{G}$, it is found here that an exponent of $m=1.0$ in Eq. (18) is adequate (see Fig. 2). The calibration parameters $\rho_{1}^{o \prime}, a_{1}^{\prime}\left(M_{I}\right)$, and $b_{1}^{\prime}\left(M_{I}\right)$, which depend on the rotational mobility, are given in Table 1 for various intermediate values of $H_{1}$ and low Zeeman modulation frequency. Corresponding calibrations for $H_{1}=0.5 \mathrm{G}$ can be found in [2].

\subsection{Two different spin labels in a lipid membrane}

Experimentally, we studied the lipid system: TEMPOtearate (TS) and 16-PCSL incorporated together in fluid DMPC bilayer membranes (see Fig. 3). These two spin labels are located in the polar and hydrophobic regions of the DMPC membrane, respectively. For this reason, and because of the different structures of the reporter group (TEMPO-based and DOXYL-based), they have different isotropic hyperfine coupling constants. As seen from Fig. 3, the presence of two EPR signals is observed already in the conventional $V_{1}$-spectrum with $100 \mathrm{kHz}$ field modulation (dotted lines), although spectral resolution in this case is less than optimum. In order to increase the difference in $T_{1}$ between the two spin labels in the $V_{1}^{\prime}$-mode, paramagnetic chromium oxalate was added to the aqueous phase at a final concentration of $50 \mathrm{mM}$ (see, e.g., [18]). It is seen that the best separation of components in the out-of-phase $V_{1}^{\prime}$-spectrum is observed at moderate microwave saturation $\left(H_{1} \sim 0.18 \mathrm{G}\right)$, in agreement with the theoretical predictions made above. A study of the dependence of the $V_{1}^{\prime}$-lineshape on Zeeman modulation frequency $\left(\omega_{m}\right)$ showed that the efficiency of signal separation increases with decreasing $\omega_{m}$ (compare solid and dashed lines in Fig. 3), again in accordance with theoretical considerations. The optimum modulation frequency, which is a compromise between the signal intensity and separation efficiency, is in the region of $\omega_{m} /$ $2 \pi=20-30 \mathrm{kHz}$. 
Table 1

$T_{1}$-calibration of the out-of-phase/in-phase $M_{I}=+1$ amplitude ratios by parameters fitting Eq. (18) (with $\left.m=1.0\right)$ at intermediate microwave fields, $H_{1}$ and Zeeman modulation frequencies, $\omega_{m}$, for rotational frequencies in the range: $f_{\mathrm{R}}=10^{5}-10^{10} \mathrm{~s}^{-1 \mathrm{a}}$

\begin{tabular}{|c|c|c|c|c|c|}
\hline$f_{\mathrm{R}}\left(\mathrm{s}^{-1}\right)$ & $H_{1}(\mathrm{G})$ & $\omega_{m} / 2 \pi(\mathrm{kHz})$ & $\rho_{1}^{o \prime}\left(M_{I}=+1\right)$ & $a_{1}^{\prime}\left(M_{I}=+1\right)$ & $b_{1}^{\prime}\left(M_{I}=+1\right)$ \\
\hline $10^{5}$ & 0.36 & 20 & -0.0117 to -0.0128 & 0.0524 to 0.0472 & 0.02875 to 0.0111 \\
\hline $10^{6}$ & 0.36 & 20 & -0.009 & 0.0343 & -0.0168 \\
\hline $10^{7}$ & 0.36 & 20 & -0.01397 & 0.0358 & -0.02747 \\
\hline $10^{8}$ & 0.36 & 20 & -0.0155 & 0.0464 & -0.0054 \\
\hline $10^{9}$ & 0.36 & 20 & -0.0228 & 0.08 & 0.01173 \\
\hline $10^{10}$ & 0.36 & 20 & -0.0224 & 0.084 & 0.0147 \\
\hline $10^{5}$ & 0.26 & 20 & -0.01173 to -0.0114 & 0.04263 to 0.0353 & 0.0196 to -0.0022 \\
\hline $10^{6}$ & 0.26 & 20 & -0.00754 & 0.024 & -0.0244 \\
\hline $10^{7}$ & 0.26 & 20 & -0.01 & 0.023 & -0.0383 \\
\hline $10^{8}$ & 0.26 & 20 & -0.0127 & 0.03242 & -0.022 \\
\hline $10^{9}$ & 0.26 & 20 & -0.02158 & 0.06267 & -0.00745 \\
\hline $10^{10}$ & 0.26 & 20 & -0.022 & 0.0687 & -0.0017 \\
\hline $10^{5}$ & 0.26 & 30 & -0.0185 & 0.0671 & 0.0604 \\
\hline $10^{6}$ & 0.26 & 30 & -0.011 & 0.037 & 0.02086 \\
\hline $10^{7}$ & 0.26 & 30 & -0.01349 & 0.03398 & -0.0387 \\
\hline $10^{8}$ & 0.26 & 30 & -0.01781 & 0.04869 & 0.01495 \\
\hline $10^{9}$ & 0.26 & 30 & -0.03171 & 0.09588 & 0.02566 \\
\hline $10^{10}$ & 0.26 & 30 & -0.03312 & 0.1063 & 0.03227 \\
\hline $10^{5}$ & 0.18 & 20 & -0.0098 & 0.0299 & 0.00388 \\
\hline $10^{6}$ & 0.18 & 20 & -0.00517 & 0.01422 & -0.03396 \\
\hline $10^{7}$ & 0.18 & 20 & -0.0062 & 0.01282 & -0.04796 \\
\hline $10^{8}$ & 0.18 & 20 & -0.00838 & 0.0191 & -0.03837 \\
\hline $10^{9}$ & 0.18 & 20 & 0.01726 & 0.0423 & -0.02654 \\
\hline $10^{10}$ & 0.18 & 20 & -0.01879 & 0.0489 & 0.01986 \\
\hline
\end{tabular}

${ }^{\mathrm{a}}$ Results are given for an intrinsic linewidth $\Delta H_{0}=1.0 \mathrm{G}$, and a peak-to-peak inhomogeneous linewidth of $1.4 \mathrm{G}$. Where a second value is given, this corresponds to $\Delta H_{0}=2.0 \mathrm{G}$. Values of $a_{1}^{\prime}\left(M_{I}=+1\right)$ and $b_{1}^{\prime}\left(M_{I}=+1\right)$ are given for $T_{1}$ in $\mu$ s.

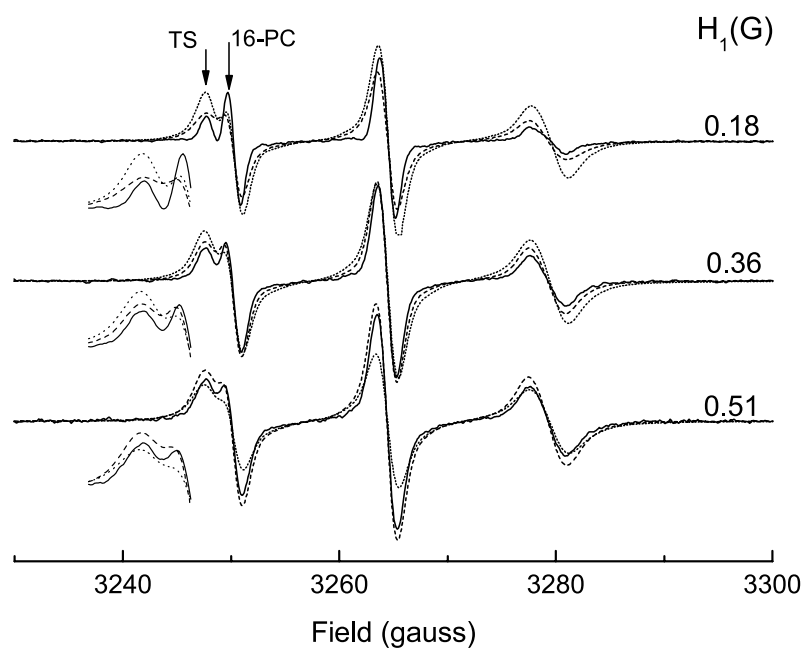

Fig. 3. First harmonic in-phase $\left(V_{1}\right)$ and out-of-phase $\left(V_{1}^{\prime}\right)$ EPR spectra of TEMPOstearate (TS) and 16-PCSL (16-PC) in DMPC membranes at $37^{\circ} \mathrm{C}$, with different Zeeman modulation frequencies and different microwave powers ( $H_{1}$ in gauss). The spin label/lipid molar ratios are $0.5 \%$ for both spin labels. Solid lines and dashed lines are $V_{1}^{\prime}$-spectra at $\omega_{m} / 2 \pi=20$ and $100 \mathrm{kHz}$, respectively, and dotted lines are $V_{1}$-spectra at $\omega_{m} / 2 \pi=100 \mathrm{kHz}$. Aqueous phase contains $50 \mathrm{mM}$ chromium oxalate. Left-hand insets are expanded versions of the low-field $\left(M_{I}=+1\right)$ positive peaks.

In fact, the $V_{1}^{\prime}$ - and $V_{1}$-modes are complementary to one other. Using the out-of-phase mode one can observe preferentially the EPR component with longer $T_{1}$. On the other hand, the spin-label EPR signal with shorter $T_{1}$ relatively has a greater amplitude in the $V_{1}$-mode, where the component with longer $T_{1}$ is preferentially saturated (compare solid and dotted lines in Fig. 3).

Values of the out-of-phase to in-phase amplitude ratios, $\rho_{1}^{\prime}(+1)$, were determined for those of the spectra in Fig. 3 for which the low-field lines were sufficiently well resolved. By using calibrations of the type given in Section 4.1, but with allowance for anisotropic rotation [5], mean values of the effective spin-lattice relaxation time are: $T_{1}=0.45 \pm 0.03$ and $0.61 \pm 0.04 \mu$ s for TEMPOstearate and 14-PCSL, respectively (in the presence of $50 \mathrm{mM}$ chromium oxalate).

\subsection{Lipid phase separation in two-component membranes}

Two-component DMPC/DSPC membranes were investigated in the region of gel-fluid phase separation, at a molar ratio of 2:3 [19]. Three phospholipid spin labels, 16-PCSL, 14-PCSL, and 6-PCSL, were used. To detect possible spectral heterogeneity, the $V_{1}^{\prime}$-EPR spectra of these spin labels were studied at different temperatures $\left(32-48^{\circ} \mathrm{C}\right) . V_{1}$ - and $V_{1}^{\prime}$-EPR spectra of 16-PCSL in DMPC/DSPC membranes at $32{ }^{\circ} \mathrm{C}$, with and without $10 \mathrm{mM} \mathrm{Ni}\left(\mathrm{ClO}_{4}\right)_{2}$, are given in Figs. $4 \mathrm{~B}$ and $\mathrm{A}$, respectively, for different microwave field strengths $H_{1}$ and different modulation frequencies $\omega_{m} . \mathrm{Ni}^{2+}$ ions have long been used as paramagnetic quenchers of aqueous spin 
A

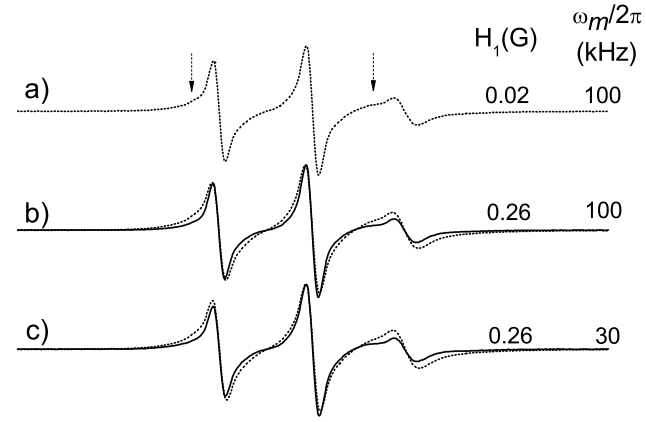

B

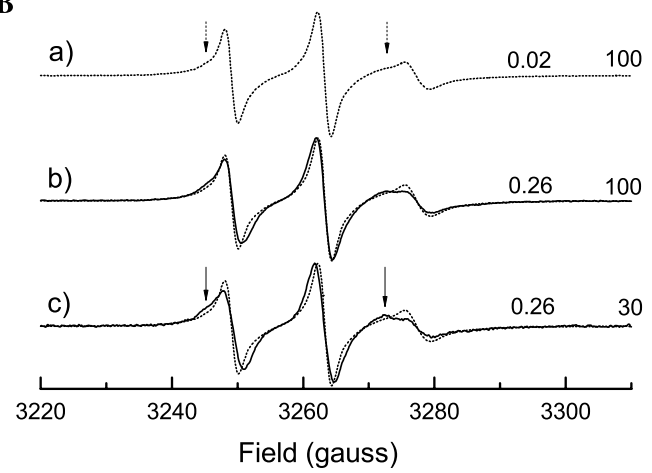

Fig. 4. First harmonic in-phase ( $V_{1}$, dotted lines) and out-of-phase $\left(V_{1}^{\prime}\right.$, solid lines) EPR spectra of 16-PCSL in $(3: 2 \mathrm{~mol} / \mathrm{mol}) \mathrm{DSPC} /$ DMPC membranes in the presence (B) and in the absence (A) of $10 \mathrm{mM} \mathrm{Ni}\left(\mathrm{ClO}_{4}\right)_{2}$ at $32{ }^{\circ} \mathrm{C}$. Spectra are recorded at the microwave amplitudes and Zeeman modulation frequencies indicated: (a) $V_{1}$-spectrum at $H_{1}=0.02 \mathrm{G}$ and $\omega_{m} / 2 \pi=100 \mathrm{kHz}$, (b) $V_{1}^{\prime}$ - and $V_{1^{-}}$ spectrum at $H_{1}=0.26 \mathrm{G}$ and $\omega_{m} / 2 \pi=100 \mathrm{kHz}$, and (c) $V_{1}^{\prime}$ - and $V_{1}$-spectrum at $H_{1}=0.26 \mathrm{G}$ and $\omega_{m} / 2 \pi=30 \mathrm{kHz}$. The positions of the unresolved low-field shoulders are indicated by the dashed arrows, and those of the resolved peaks by the solid arrows.

labels (see, e.g., [20]), and $\mathrm{Ni}\left(\mathrm{ClO}_{4}\right)_{2}$ is a particularly effective relaxant for membrane-embedded spin labels [21]. As seen from spectrum (a) in Figs. 4A and B, the conventional low-power $V_{1}$-EPR spectra (dotted lines) are dominated by the sharp, mobile component, both in the presence and in the absence of nickel perchlorate. Only the broad shoulders to low-field of the $M_{I}=-1$ and +1 hyperfine lines give a hint of the presence of a second, less mobile species (indicated by dotted arrows). In the absence of $\mathrm{Ni}\left(\mathrm{ClO}_{4}\right)_{2}$, the out-of-phase $V_{1}^{\prime}$-spectrum (solid line) is narrower than the $V_{1}$-spectrum (dotted line), as predicted by theory, but also does not consist very clearly of two spectral components [see spectra (b) and (c) of Fig. 4A]. However, in the presence of $\mathrm{Ni}\left(\mathrm{ClO}_{4}\right)_{2}$, a second $V_{1}^{\prime}$-EPR signal is evident that corresponds to more strongly immobilized spin labels [solid-line spectra (b) and (c) in Fig. 4B]. It can be seen that, along with the new low-field maximum, another maximum appears in the high-field region at a position corresponding to the resonance absorption for spin labels oriented perpendicular to the magnetic field (indicated by solid arrows in both regions). As in the case of the two-label system described above, maximum spectral resolution of the less mobile component is observed at lower Zeeman modulation frequency $(30 \mathrm{kHz})$ and moderate microwave saturation $\left(H_{1}=0.26 \mathrm{G}\right)$. This is given by the solid-line spectrum (c) in Fig. 4B.

Although the absolute intensity of the $V_{1}^{\prime}$-spectrum decreases in the presence of $\mathrm{Ni}\left(\mathrm{ClO}_{4}\right)_{2}$ (see Table 2), the relative intensities of the two out-of-phase EPR components become comparable (see Fig. 4B). This is expected if the spin relaxation enhancement induced by $\mathrm{Ni}^{2+}$ ions is much less for the more strongly immobilized component than for the weakly immobilized component. Therefore, the gel-phase membrane domains which are responsible for the more immobilized EPR spectrum are less permeable to nickel perchlorate than are the fluid-phase domains. For the fluid component, the $\rho_{1}^{\prime}(+1)$ out-ofphase/in-phase amplitude ratios of 16-PCSL at $32{ }^{\circ} \mathrm{C}$ yield an enhancement in effective spin-lattice relaxation rate of $1.5-1.7 \times 10^{6} \mathrm{~s}^{-1}$ by $10 \mathrm{mM} \mathrm{Ni(-}$ $\left.\mathrm{ClO}_{4}\right)_{2}$, from measurements at modulation frequencies of both 30 and $100 \mathrm{kHz}$.

Two-component lineshape simulations were made for the solid-line $V_{1}^{\prime}$-EPR spectra in Figs. 4B, (b) and (c) by using pseudo-powder patterns with the anisotropy defined by an order parameter $\left(S_{z z}\right)$, and allowing for anisotropy in the $T_{1}$-relaxation. These simplified simulations yield a value of 0.55 for the ratio of gel to fluid populations of spin-labelled lipid, where the effective $T_{1}$ of $16-\mathrm{PCSL}$ is approximately $2 \mu \mathrm{s}$ in the gel phase and $0.5-0.6 \mu \mathrm{s}$ in the fluid phase. The corresponding order parameters of 16-PCSL are $S_{\text {gel }}=0.48$ and $S_{\text {fluid }}=0.1$ in the gel and fluid phases, respectively, at $32{ }^{\circ} \mathrm{C}$.

At the higher temperature of $45^{\circ} \mathrm{C}$, the $V_{1}^{\prime}$-spectrum and also the corresponding $V_{1}$-spectrum of 16-PCSL do not explicitly reveal the presence of two spectral components, even when using a modulation frequency of $\omega_{m} / 2 \pi=30 \mathrm{kHz}$ (see Fig. 5B). However, the linewidths of the out-of-phase $V_{1}^{\prime}$-hyperfine components (solid line) are broader than those of the in-phase $V_{1}$-spectra (dotted line), in contrast to what is found from spectral

Table 2

Out-of-phase/in-phase ratios, $\rho_{1}^{\prime}\left(M_{I}\right)$, for the $V_{1}^{\prime} / V_{1}$ EPR amplitudes of 14-PCSL in DMPC/DSPC $(2: 3 \mathrm{~mol} / \mathrm{mol})$ membranes in the presence and absence of $20 \mathrm{mM} \mathrm{Ni}\left(\mathrm{ClO}_{4}\right)_{2}{ }^{\mathrm{a}}$

\begin{tabular}{|c|c|c|c|}
\hline Medium & $T\left({ }^{\circ} \mathrm{C}\right)$ & $\rho_{1}^{\prime}(+1)$ & $\rho_{1}^{\prime}(0)$ \\
\hline Water & 21 & 0.194 & 0.285 \\
\hline $20 \mathrm{mM} \mathrm{Ni}\left(\mathrm{ClO}_{4}\right)_{2}$ & 21 & 0.015 & 0.015 \\
\hline $20 \mathrm{mM} \mathrm{Ni}\left(\mathrm{ClO}_{4}\right)_{2}$ & 31 & 0.013 & 0.013 \\
\hline $20 \mathrm{mM} \mathrm{Ni}\left(\mathrm{ClO}_{4}\right)_{2}$ & 41 & 0.016 & 0.016 \\
\hline $20 \mathrm{mM} \mathrm{Ni}\left(\mathrm{ClO}_{4}\right)_{2}$ & 48 & 0.017 & 0.019 \\
\hline
\end{tabular}




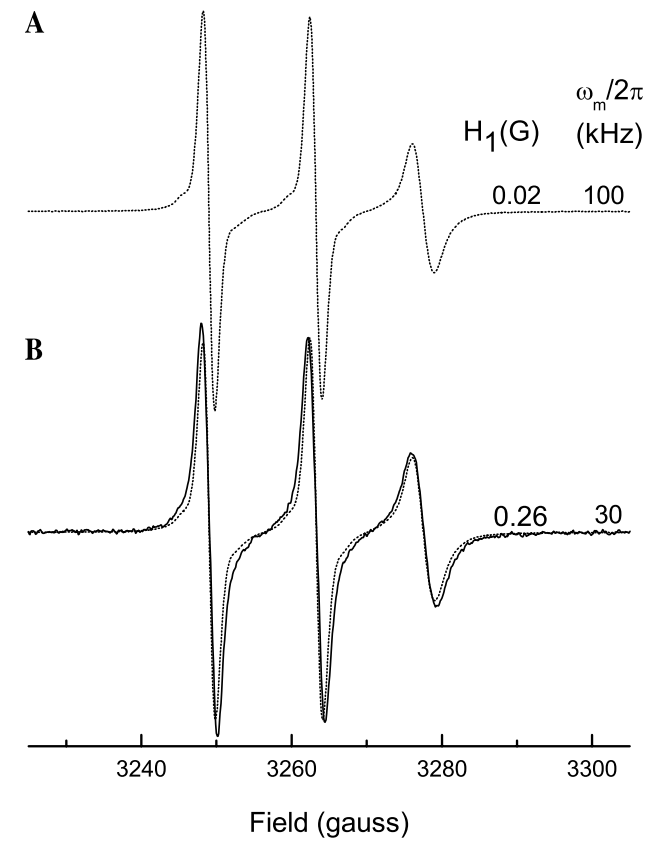

Fig. 5. $V_{1^{-}}$(dotted line) and $V_{1}^{\prime}$ - (solid line) EPR spectra of 16-PCSL in $3: 2 \mathrm{~mol} / \mathrm{mol}$ DSPC/DMPC membranes in the presence of $10 \mathrm{mM}$ $\mathrm{Ni}\left(\mathrm{ClO}_{4}\right)_{2}$ at $45^{\circ} \mathrm{C}$. (A) $V_{1}$-spectrum at $H_{1}=0.02 \mathrm{G}$ and $\omega_{m} /$ $2 \pi=100 \mathrm{kHz},(\mathrm{B}) V_{1}^{\prime}-$ and $V_{1^{-}}$-spectrum at $H_{1}=0.26 \mathrm{G}$ and $\omega_{m} /$ $2 \pi=30 \mathrm{kHz}$.

simulations for one-component systems (see Section 2 and Fig. 1). Therefore, the greater broadening of the $V_{1}^{\prime}$-spectra is indicative of a heterogeneity of the spin label environment in DSPC/DMPC membranes in the phase separation region. The lack of explicit separation in this case may be because both EPR signals are in the rapid motion region, and therefore their overlap results only in broadening of all three hyperfine components. Two-component simulations for Fig. 5 yield a ratio of gel to fluid populations of 0.45 , and effective $T_{1}$-relaxation times of ca. 2 and $0.5 \mu \mathrm{s}$, respectively. In this case, the gel and fluid components are distinguished solely by their $T_{1}$-values, because the order parameters have a common low value of 0.05 for both components.

A situation similar to that for 16-PCSL at $45^{\circ} \mathrm{C}$ occurs also for the 14-PCSL spin label, the EPR spectra of which are given in Fig. 6. It is seen that at both 41 and $48{ }^{\circ} \mathrm{C}$ the linewidths of the $V_{1}^{\prime}$-spectra (solid lines) from 14-PCSL are broader than for the corresponding $V_{1}$-spectra (dotted lines). On the other hand, in the gel phase at $21^{\circ} \mathrm{C}$, in the absence of paramagnetic relaxant, the linewidth of the out-of-phase $V_{1}^{\prime}$-spectrum is less than for the in-phase $V_{1}$-spectrum (see solid and dotted lines, respectively, in Fig. 6B), as expected for a singlecomponent EPR signal. The latter result indicates that one component dominates at this lower temperature. Similar findings are also obtained with 6-PCSL: on the one hand for membranes in $20 \mathrm{mM} \mathrm{Ni}\left(\mathrm{ClO}_{4}\right)_{2}$ at $45^{\circ} \mathrm{C}$, and on the other hand for membranes in water at $21{ }^{\circ} \mathrm{C}$ (spectra not shown).
A
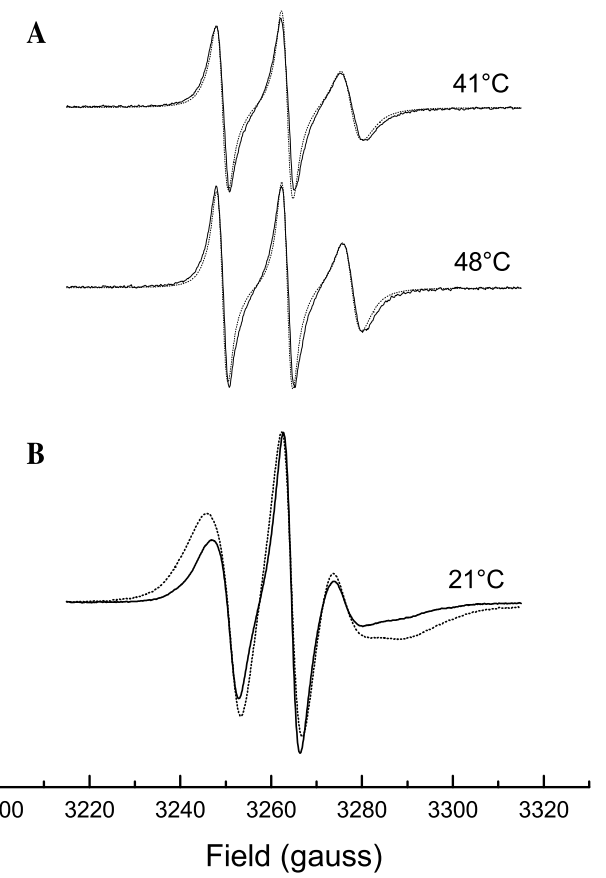

Fig. 6. $V_{1}$ - (dotted line) and $V_{1}^{\prime}$ - (solid line) EPR spectra of 14-PCSL in $3: 2 \mathrm{~mol} / \mathrm{mol} \mathrm{DSPC} / \mathrm{DMPC}$ membranes. (A) In the presence of $10 \mathrm{mM} \mathrm{Ni}\left(\mathrm{ClO}_{4}\right)_{2}$ at 41 and $48{ }^{\circ} \mathrm{C}$, as indicated, and (B) dispersed in water at $21{ }^{\circ} \mathrm{C}$. $H_{1}=0.51 \mathrm{G}$ and $\omega_{m} / 2 \pi=100 \mathrm{kHz}$.

Thus, qualitatively consistent results on the phase separation behavior are obtained with three spin labels located at different depths in the membrane.

\subsection{Lipid-peptide complexes: interaction of gramicidin A with DMPC membranes}

In previous studies [22-25], mostly on membranes with rather high gramicidin A contents (GA/lipid $\geqslant 0.1 \mathrm{~mol} / \mathrm{mol}$ ), it was found that the conventional low-power EPR absorption spectra of phospholipids spin-labelled close to the terminal methyl group of the $s n-2$ chain contain two components. The more motionally restricted component $(b)$ is usually assigned to the boundary lipids contacting the GA aggregates [22-25], in analogy with similar studies on integral transmembrane proteins [26]. The weakly immobilized component (f) corresponds to the fluid (free) lipids, although the EPR spectra of the latter indicate considerably lower rotational mobility as compared with membranes of fluid lipids alone.

We studied the $V_{1}$ - and $V_{1}^{\prime}$-spectra of 16-PCSL in GA/DMPC membranes in order to explore the potential of out-of-phase EPR for obtaining new information on lipid dynamics in these complexes. A GA/DMPC molar ratio of 0.2 was used. As seen from Fig. 7A, the out-ofphase $V_{1}^{\prime}$-spectra (solid lines) contain two components that are already resolved in the in-phase $V_{1}$-spectra (dotted lines). The outer hyperfine splittings of the 

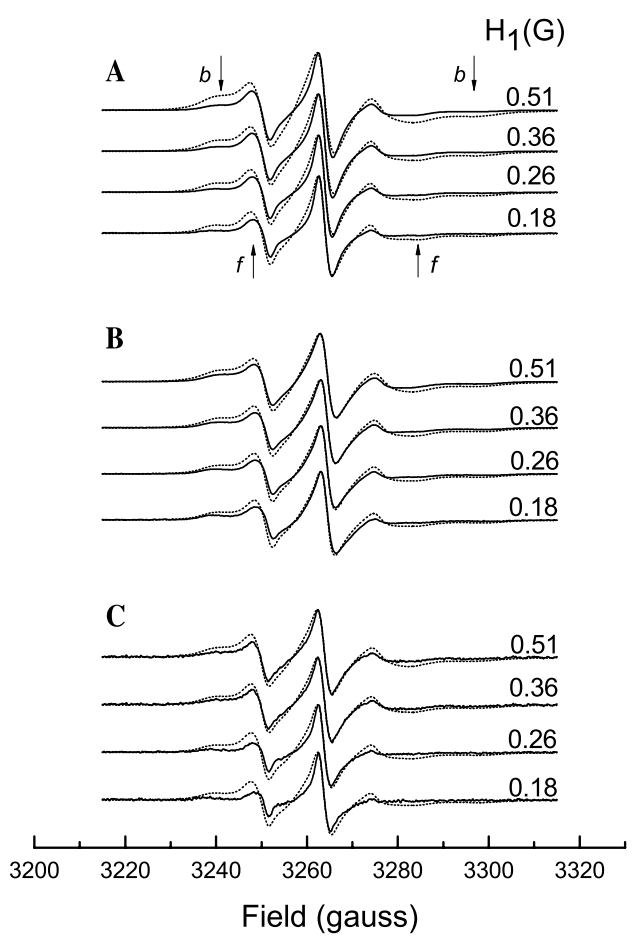

Fig. 7. $V_{1}$ - (dotted lines) and $V_{1}^{\prime}$ - (solid lines) EPR spectra of 16-PCSL in $1: 5 \mathrm{~mol} / \mathrm{mol} \mathrm{GA} / \mathrm{DMPC}$ membranes at $20^{\circ} \mathrm{C}$. The $H_{1}$-field is: 0.51 , $0.36,0.26$, and $0.18 \mathrm{G}$, as indicated in each panel. (A) Membranes in water, $\omega_{m} / 2 \pi=100 \mathrm{kHz}$. (B) Membranes in $10 \mathrm{mM} \mathrm{Ni}\left(\mathrm{ClO}_{4}\right)_{2}, \omega_{m} /$ $2 \pi=100 \mathrm{kHz}$. (C) Membranes in $10 \mathrm{mM} \mathrm{Ni}\left(\mathrm{ClO}_{4}\right)_{2}, \omega_{m} / 2 \pi=20 \mathrm{kHz}$. The positions of the outer hyperfine peaks from the motionally restricted $(b)$ and fluid $(f)$ lipid components are indicated by vertical arrows in (A).

motionally restricted $(b)$ and more weakly immobilized $(f)$ components are indicated by arrows in Fig. 7A. The effects of microwave saturation level, Zeeman modulation frequency, the paramagnetic relaxant $\mathrm{Ni}\left(\mathrm{ClO}_{4}\right)_{2}$, and temperature on the $V_{1}$ - and $V_{1}^{\prime}$-spectra were studied. In contrast to DSPC/DMPC membranes, the lineshapes of the in-phase and out-of-phase spectral modes do not change greatly under these various conditions (see Figs. $7 \mathrm{~A}-\mathrm{C}$ ), although the spectral resolution between the two low-field components increases substantially in the $V_{1}^{\prime}$-mode on decreasing the microwave field intensity $H_{1}$ and the Zeeman modulation frequency $\omega_{m}$.

\subsubsection{Motional heterogeneity in the lipids associated with $G A$}

Closer inspection of Fig. 7 shows that the separations $\left(2 A_{z z}^{\prime}\right)$ of the outer extrema from the motionally restricted component are not identical for $V_{1}^{\prime}$ - and $V_{1}$-spectra measured at the same $H_{1}$-field. The value of $2 A_{z z}^{\prime}$ is always greater for the $V_{1}^{\prime}$-spectrum than for the $V_{1}$-spectrum. Moreover, the value of $2 A_{z z}^{\prime}$ increases with decreasing $H_{1}$, for both the $V_{1^{-}}$and $V_{1}^{\prime}$-spectra (see Table 3). In contrast, simulations of single-component EPR spectra for the motionally restricted spin labels show that the outer extrema of the $V_{1}$ - and $V_{1}^{\prime}$-spectra
Table 3

Separation of the outer extrema $\left(2 A_{z z}^{\prime}\right)$ of the motionally restricted component in the in-phase $\left(V_{1}\right)$ and out-of-phase $\left(V_{1}^{\prime}\right)$ EPR spectra from 16-PCSL in GA: DMPC $(1: 5 \mathrm{~mol} / \mathrm{mol})$ membranes at different microwave fields $\left(H_{1}\right)$, concentrations $\left(c_{\mathrm{Ni}}\right)$ of $\mathrm{Ni}\left(\mathrm{ClO}_{4}\right)_{2}$, and modulation frequencies $\left(\omega_{m}\right)$, at $20^{\circ} \mathrm{C}$

\begin{tabular}{lllll}
\hline$\omega_{m} / 2 \pi(\mathrm{kHz})$ & $c_{\mathrm{Ni}}(\mathrm{mM})$ & $H_{1}(\mathrm{G})$ & $2 A_{z z}^{\prime}\left(V_{1}\right)(\mathrm{G})$ & $2 A_{z z}^{\prime}\left(V_{1}^{\prime}\right)(\mathrm{G})$ \\
\hline 100 & 0 & 0.51 & 55.3 & 58.6 \\
100 & 0 & 0.36 & 56.5 & 58.5 \\
100 & 0 & 0.26 & 57.5 & 59.5 \\
100 & 0 & 0.18 & 57.8 & 61.9 \\
100 & 10 & 0.51 & 55.3 & 57.1 \\
100 & 10 & 0.36 & 56.6 & 59.0 \\
100 & 10 & 0.26 & 57.4 & 61.1 \\
100 & 10 & 0.18 & 58.2 & 62.1 \\
20 & 10 & 0.26 & 57.1 & 60.9 \\
20 & 10 & 0.18 & 58.8 & 64.0 \\
\hline
\end{tabular}

corresponding to different order parameters $\left(S_{z z}\right)$ coincide with rather high accuracy, and further that $2 A_{z z}^{\prime}$ does not depend on the $H_{1}$-field. The differences in $2 A_{z z}^{\prime}$ between the $V_{1^{-}}$and $V_{1}^{\prime}$-spectra, and the dependence of $2 A_{z z}^{\prime}$ on $H_{1}$, are therefore indicative of heterogeneity in the population of motionally restricted spin-labelled chains at the lipid-peptide interface.

The distribution in $2 A_{z z}^{\prime}$ most probably arises from differences in rotational mobility (rotational frequencies or/and order parameters) within the interfacial population of spin labels. In the linear $V_{1}$-spectrum, this distribution is not observed directly because it contributes only to inhomogeneous broadening of the $M_{I}= \pm 1$ hyperfine components. If, however, the spin labels with different rotational mobility have different spin-lattice relaxation times, then under saturation conditions the contribution from spin labels with shorter $T_{1}$ will dominate in the outer extrema of the $V_{1}$-spectra, because of saturation of the components with longer $T_{1}$. On the other hand, the contribution to the outer extrema from spin labels with longer $T_{1}$ will dominate in the $V_{1}^{\prime}$-spectrum. Therefore, the positions of the outer extrema will not coincide in the $V_{1^{-}}$and $V_{1}^{\prime}$-spectra. That longer $T_{1}$ relaxation times correspond to larger values of $2 A_{z z}^{\prime}$ may reasonably be expected for contributions from molecular motions to the spin-lattice relaxation rate. A decrease in either the rotational amplitude or frequency results in a decrease in spin-lattice relaxation rate and in an increase in the effective values of $2 A_{z z}^{\prime}$. The observation of a distribution in $T_{1}$ (and $2 A_{z z}^{\prime}$ ) implies that the life time of the distribution is greater than the mean spin-lattice relaxation time (i.e., is greater than $1 \mu \mathrm{s})$. It is found from Fig. 7 that the difference in $2 A_{z z}^{\prime}$ between the $V_{1^{-}}$and $V_{1}^{\prime}$-spectra is observed clearly at $20^{\circ} \mathrm{C}$, both in the presence and absence of $10 \mathrm{mM}$ $\mathrm{Ni}\left(\mathrm{ClO}_{4}\right)_{2}$. However, the difference decreases at $30^{\circ} \mathrm{C}$, and in the presence of $30 \mathrm{mM} \mathrm{Ni}\left(\mathrm{ClO}_{4}\right)_{2}$. Probably, at the higher temperature and higher concentration of paramagnetic relaxant, the different $T_{1}$ relaxation rates 
within the population of rotationally restricted spin labels become motionally averaged.

Characterization of the motional distribution is afforded by the values of $2 A_{z z}^{\prime}$ from $V_{1}$ - and $V_{1}^{\prime}$-spectra measured at different $H_{1}$-fields that are given in Table 3. The difference in $2 A_{z z}^{\prime}$ between $V_{1}$ - and $V_{1}^{\prime}$-spectra increases, and is seen more clearly, at lower $H_{1}$-fields. Additionally, the absolute values of $2 A_{z z}^{\prime}$ increase with decreasing microwave saturation, for both $V_{1}$ - and $V_{1}^{\prime}$-spectra. If the difference in $2 A_{z z}^{\prime}$ is attributed solely to ordering of the lipid spin labels, then the maximum difference in $2 A_{z z}^{\prime}$ (from 55.3 to $64 \mathrm{G}$, see Table 3 ) corresponds to a distribution in order parameter from $S_{z z}=0.63-0.85$, at $20{ }^{\circ} \mathrm{C}$. The corresponding distribution in $T_{1}$-relaxation times was estimated from the values of $\rho_{1}^{\prime}(+1)$ measured at field positions corresponding to the low-field maxima of the respective $V_{1}$ - and $V_{1}^{\prime}$-spectra. These values of $\rho_{1}^{\prime}(+1)$ and the effective $T_{1}$-relaxation times that are deduced from them are given in Table 4 . For samples at $30{ }^{\circ} \mathrm{C}$ in the presence of $30 \mathrm{mM} \mathrm{Ni}\left(\mathrm{ClO}_{4}\right)_{2}$, only single values are presented, because the differences in $\rho_{1}^{\prime}(+1)$ and $T_{1}$ at the two field positions are rather small. Nevertheless, the data in Table 4 demonstrate the potential of non-linear $V_{1}^{\prime}$ - and $V_{1}$-EPR spectra for defining the motional heterogeneity of the GA-associated lipids. It is also seen from Table 4 that $T_{1}$-relaxation times determined at different $H_{1}$-fields are, in general, close to one other, although a progressive increase in $T_{1}$ with decreasing $H_{1}$-field is observed. This tendency also can be explained by a heterogeneity in the motionally restricted lipid species, because at low $H_{1}$-fields the outer extrema tend to be dominated by species with higher values of $2 A_{z z}^{\prime}$, and hence with longer $T_{1}$.

\subsubsection{Rotational mobility of GA-associated lipids}

Table 4 summarizes the out-of-phase to in-phase amplitude ratios $\rho_{1}^{\prime}(b)$ and $\rho_{1}^{\prime}(f)$ measured for the low-field $\left(M_{I}=+1\right)$ components of both the motionally restricted $(b)$ and fluid $(f)$ signals, respectively, at different values of $H_{1}$ and $\omega_{m}$, different concentrations of $\mathrm{Ni}\left(\mathrm{ClO}_{4}\right)_{2}$, and different temperatures. The amplitude ratios $h_{b} / h_{f}$ of the motionally restricted to fluid

Table 4

Out-of-phase/in-phase ratios, $\rho_{1}^{\prime}\left(M_{I}=+1\right)$, of the motionally restricted $(b)$ and fluid $(f)$ low-field components, the corresponding effective spin-lattice relaxation times, $T_{1 b}$ and $T_{1 f}$, and the amplitude ratios $\left(h_{b} / h_{f}\right)$ of the two components in the in-phase $\left(V_{1}\right)$ and out-of-phase $\left(V_{1}^{\prime}\right)$ EPR spectra for 16-PCSL in GA: DMPC $(1: 5 \mathrm{~mol} / \mathrm{mol})$ membranes in the presence of different concentrations, $c_{\mathrm{Ni}}$, of $\mathrm{Ni}\left(\mathrm{ClO}_{4}\right)_{2}{ }^{\mathrm{a}}$

\begin{tabular}{|c|c|c|c|c|c|c|c|c|c|}
\hline$\omega_{m} / 2 \pi(\mathrm{kHz})$ & $T\left({ }^{\circ} \mathrm{C}\right)$ & $c_{\mathrm{Ni}}(\mathrm{mM})$ & $H_{1}(\mathrm{G})$ & $\rho_{1}^{\prime}(b)$ & $T_{1 b}(\mu \mathrm{s})$ & $\rho_{1}^{\prime}(f)$ & $T_{1 f}(\mu \mathrm{s})$ & $\left(h_{b} / h_{f}\right)_{V_{1}}$ & $\left(h_{b} / h_{f}\right)_{V_{1}^{\prime}}$ \\
\hline \multirow[t]{2}{*}{100} & 20 & 0 & 0.51 & 0.09 & 0.77 & 0.16 & 0.78 & 0.55 & 0.28 \\
\hline & & & & 0.094 & 0.8 & & & & \\
\hline \multirow[t]{2}{*}{100} & 20 & 0 & 0.36 & 0.078 & 0.98 & 0.138 & 0.94 & 0.47 & 0.26 \\
\hline & & & & 0.08 & 1.0 & & & & \\
\hline \multirow[t]{2}{*}{100} & 20 & 0 & 0.26 & 0.049 & 1.0 & 0.085 & 0.94 & 0.41 & 0.24 \\
\hline & & & & 0.051 & 1.1 & & & & \\
\hline \multirow[t]{2}{*}{100} & 20 & 0 & 0.18 & 0.025 & 1.0 & 0.046 & 0.99 & 0.39 & 0.21 \\
\hline & & & & 0.027 & 1.1 & & & & \\
\hline 100 & 20 & 0 & 0.02 & & & & & 0.36 & \\
\hline \multirow[t]{2}{*}{20} & 20 & 0 & 0.51 & 0.035 & 1.1 & 0.05 & 0.96 & 0.49 & 0.35 \\
\hline & & & & 0.036 & 1.1 & & & & \\
\hline \multirow[t]{2}{*}{20} & 20 & 0 & 0.36 & 0.026 & 1.1 & 0.04 & 1.0 & 0.47 & 0.30 \\
\hline & & & & 0.027 & 1.2 & & & & \\
\hline \multirow[t]{2}{*}{20} & 20 & 0 & 0.26 & 0.016 & 1.1 & 0.025 & 1.0 & 0.42 & 0.24 \\
\hline & & & & 0.017 & 1.2 & & & & \\
\hline \multirow[t]{2}{*}{100} & 20 & 10 & 0.51 & 0.1 & 0.86 & 0.144 & 0.71 & 0.48 & 0.36 \\
\hline & & & & 0.108 & 0.88 & & & & \\
\hline \multirow[t]{2}{*}{100} & 20 & 10 & 0.36 & 0.054 & 0.76 & 0.107 & 0.77 & 0.47 & 0.34 \\
\hline & & & & 0.056 & 0.78 & & & & \\
\hline \multirow[t]{2}{*}{100} & 20 & 10 & 0.26 & 0.034 & 0.81 & 0.065 & 0.79 & 0.45 & 0.35 \\
\hline & & & & 0.036 & 0.85 & & & & \\
\hline 100 & 20 & 30 & 0.51 & 0.084 & 0.74 & 0.098 & 0.56 & 0.48 & \\
\hline 100 & 20 & 30 & 0.36 & 0.057 & 0.8 & 0.067 & 0.59 & & \\
\hline 100 & 20 & 30 & 0.26 & 0.034 & 0.82 & 0.041 & 0.62 & 0.43 & 0.36 \\
\hline 100 & 20 & 30 & 0.18 & 0.02 & 0.91 & 0.022 & 0.67 & 0.41 & 0.38 \\
\hline 100 & 24 & 30 & 0.36 & 0.056 & 0.77 & 0.063 & 0.55 & & \\
\hline 100 & 24 & 30 & 0.26 & 0.036 & 0.83 & 0.039 & 0.58 & 0.3 & 0.28 \\
\hline 100 & 30 & 30 & 0.51 & 0.053 & 0.57 & 0.063 & 0.40 & 0.26 & 0.20 \\
\hline 100 & 30 & 30 & 0.36 & 0.042 & 0.64 & 0.046 & 0.45 & & \\
\hline 100 & 30 & 30 & 0.26 & 0.026 & 0.69 & 0.027 & 0.48 & 0.20 & 0.19 \\
\hline 100 & 30 & 30 & 0.02 & & & & & 0.18 & \\
\hline
\end{tabular}

${ }^{\text {a }} \omega_{m}$ is the Zeeman modulation frequency, $H_{1}$ is the microwave field intensity, and $T$ is the temperature. The two values of $\rho_{1}^{\prime}(b)$ and $T_{1 b}$ correspond to evaluations of the $V_{1}$ - and $V_{1}^{\prime}$-amplitudes at the positions of the low-field maxima of the $V_{1}$ - and $V_{1}^{\prime}$-spectra, respectively. 
components for the $M_{I}=+1$ manifold of both the $V_{1^{-}}$ and $V_{1}^{\prime}$-modes are also given in Table 4.

Simulations of the $V_{1^{-}}$and $V_{1}^{\prime}$-spectra that are presented in Fig. 7A, and also the corresponding $\rho_{1}^{\prime}(+1)$ ratios, show that, in the absence of $\mathrm{Ni}\left(\mathrm{ClO}_{4}\right)_{2}$, the effective $T_{1}$-relaxation times for both motionally restricted and fluid components are approximately equal. These values of $T_{1}(\approx 1 \mu \mathrm{s})$ are listed in Table 4. However, it is also seen from Table 4 that the outof-phase amplitude ratio, $\rho_{1}^{\prime}(b)$, of the motionally restricted lipid population is less than that of the fluid lipid population, $\rho_{1}^{\prime}(f)$, under all conditions without $\mathrm{Ni}\left(\mathrm{ClO}_{4}\right)_{2}$. In addition, the ratio of amplitudes of the motionally restricted to fluid lipid spectral components, $h_{b} / h_{f}$, is smaller in the $V_{1}^{\prime}$-mode than in the $V_{1}$-mode, in the absence of $\mathrm{Ni}\left(\mathrm{ClO}_{4}\right)_{2}$. These differences arise because of the non-vanishing effects of rotational motion on the first harmonic out-of-phase to in-phase $\rho_{1}^{\prime}$ amplitude ratios $[2,5]$. Rotational diffusion coefficients, $D_{\mathrm{R} \perp}$, were estimated in $[23,24]$ for GA in dipalmitoyl phosphatidylcholine $(1: 5 \mathrm{~mol} / \mathrm{mol})$ at $45^{\circ} \mathrm{C}$ to be about $0.2 \times 10^{8}$ and $2 \times 10^{8} \mathrm{~s}^{-1}$ for the motionally restricted and fluid spin-labelled lipids, respectively. The slower motion of the restricted (boundary) lipids, with rotational correlation times of $1 / 6 D_{\mathrm{R} \perp} \cong 10^{-8} \mathrm{~s}$, therefore results in much larger values of $1 / T_{2}^{\text {eff }}$ than for the fluid lipids [1] and, correspondingly, in lower values of the $\rho_{1}^{\prime}$ ratio for the same $T_{1}$-relaxation time, as is found in Table 4.

Taking a mean spin-lattice relaxation time of $T_{1}=0.9 \mu \mathrm{s}$, the $V_{1}^{\prime}$-EPR data recorded with $H_{1}=0.51 \mathrm{G}$ and $\omega_{m} / 2 \pi=100 \mathrm{kHz}$ that are given in Table 4 for samples without $\mathrm{Ni}\left(\mathrm{ClO}_{4}\right)_{2}$ yield rotational frequencies of $(1-2) \times 10^{7} \mathrm{~s}^{-1}$ for the motionally restricted component $(b)$ and $(2-3) \times 10^{8} \mathrm{~s}^{-1}$ for the fluid component $(f)$. These values are deduced from the experimental $\rho_{1}^{\prime}\left(M_{I}=+1\right)$ ratios for the low-field manifold by using the calibration parameters given in [2], and are seen to be in reasonable accord with the lipid rotational diffusion coefficients for GA in dipalmitoyl phosphatidylcholine that were quoted above $[23,24]$.

The values of the $\rho_{1}^{\prime}(b)$ and $\rho_{1}^{\prime}(f)$ ratios for samples in the absence of $\mathrm{Ni}\left(\mathrm{ClO}_{4}\right)_{2}$ decrease more than 2-3 times on reducing the modulation frequency from 100 to $20 \mathrm{kHz}$ (see Table 4). Nonetheless, the data recorded at the two modulation frequencies produce reasonably consistent estimates for the mobility of the two lipid components associated with GA. Using calibrations from [2] and taking $T_{1}=0.95 \mu \mathrm{s}$, the $\rho_{1}^{\prime}\left(M_{I}=+1\right)$ ratios recorded at the lower modulation frequency of $\omega_{m} / 2 \pi=20 \mathrm{kHz}$, again with $H_{1}=0.51 \mathrm{G}$, yield rotational frequencies of $(1-3) \times 10^{7} \mathrm{~s}^{-1}$ for the motionally restricted component $(b)$ and ca. $2 \times 10^{8} \mathrm{~s}^{-1}$ for the fluid component $(f)$. We note also that the ratio $\rho_{1}^{\prime}(b) / \rho_{1}^{\prime}(f)$, for motionally restricted and fluid lipids, increases at lower modulation frequency. This is because the dependence of the $\rho_{1}^{\prime}$ ratios on rotational motion decreases with decreasing modulation frequency [2].

\subsubsection{Paramagnetic relaxation enhancements}

In the presence of $10 \mathrm{mM} \mathrm{Ni}\left(\mathrm{ClO}_{4}\right)_{2}$ at $20^{\circ} \mathrm{C}$, the spin-lattice relaxation times of the fluid $\left(T_{1 f}\right)$ and motionally restricted $\left(T_{1 b}\right)$ components decrease moderately (see Table 4). The paramagnetic enhancements in relaxation rate are comparable for the two components: $\Delta\left(1 / T_{1}\right)=1 / T_{1}-1 / T_{1}^{o} \approx 2-3 \times 10^{5} \mathrm{~s}^{-1}$, deduced from the data of Table 4. In the presence of $30 \mathrm{mM} \mathrm{Ni}\left(\mathrm{ClO}_{4}\right)_{2}$ at $20^{\circ} \mathrm{C}$, the relaxation enhancement of the motionally restricted component changes little, whereas that of the fluid lipid component increases by a factor of almost three, to $6 \times 10^{5} \mathrm{~s}^{-1}$. If the intrinsic temperature dependence of the relaxation rate is neglected, the relaxation enhancement of the fluid component by $30 \mathrm{mM} \mathrm{Ni}(-$ $\left.\mathrm{ClO}_{4}\right)_{2}$ increases progressively to $1.1 \times 10^{6} \mathrm{~s}^{-1}$, between 20 and $30^{\circ} \mathrm{C}$. That of the motionally restricted component increases significantly only at the higher temperature, from $3 \times 10^{5} \mathrm{~s}^{-1}$ at $24{ }^{\circ} \mathrm{C}$ to $5 \times 10^{5} \mathrm{~s}^{-1}$ at $30^{\circ} \mathrm{C}$. The difference between the relaxation enhancements of the two components implies a (2-3)-fold preferential partitioning of $\mathrm{Ni}\left(\mathrm{ClO}_{4}\right)_{2}$ into the fluid lipids associated with GA, relative to the motionally restricted lipids.

\subsubsection{Conclusions on GA-lipid interactions}

The behavior of the lipids in GA/DMPC complexes differs strongly from that in DSPC/DMPC membranes, where the effect of $20 \mathrm{mM} \mathrm{Ni}\left(\mathrm{ClO}_{4}\right)_{2}$ on the EPR spectra of 14-PCSL is to decrease the values of the $\rho_{1}^{\prime}$-ratio by more than an order of magnitude (see Table 2). This difference in accessibility to the paramagnetic relaxant may be due to a rather different lipid organization in the lipid-peptide complex from that in the DSPC/DMPC membrane. Evidently, the distinction between motionally restricted and fluid lipids in the GA/DMPC complexes is very different from that between gel and fluid lipid domains in DSPC/DMPC membranes exhibiting lateral phase separation. This is not entirely surprising in view of the fact that motional restriction of the lipid chains by gramicidin arises from a local interaction with the peptide, whereas domain formation in DSPC/DMPC membranes involves highly cooperative interactions between the lipids. There is no evidence that the interaction of gramicidin A with DMPC involves any phase transformation at the lipid/peptide ratios used here [27-30].

The $\rho_{1}^{\prime}$-ratios of both the fluid and motionally restricted lipid components associated with gramicidin A decrease on increasing the temperature from 20 to $30^{\circ} \mathrm{C}$. At $30^{\circ} \mathrm{C}$, these parameters become approximately equal one to another (see Table 4), because the $\rho_{1}^{\prime}(f)$ ratio for the fluid lipids decreases more than does $\rho_{1}^{\prime}(b)$. Ignoring the smaller effects of changes in mobility on the calibration parameters in Eq. (18) (cf. [2]), an increase in effective spin-lattice relaxation rate of 
$\Delta\left(1 / T_{1}\right)=5 \times 10^{5} \mathrm{~s}^{-1}$ between 20 and $30^{\circ} \mathrm{C}$ is deduced from the $\rho_{1}^{\prime}(f)$ ratios in Table 4 , as compared with $\Delta\left(1 / T_{1}\right)=4 \times 10^{5} \mathrm{~s}^{-1}$ from the $\rho_{1}^{\prime}(b)$ ratios. The direction of this change is that expected from the dependence of liquid-state mechanisms for spin-lattice relaxation on rotational correlation time $\left(\tau_{\mathrm{R}}\right)$, where the motionally restricted component $(b)$ has the longer $\tau_{\mathrm{R}}[12,13]$. The different response of the $\rho_{1}^{\prime}(b)$ and $\rho_{1}^{\prime}(f)$ ratios to the addition of nickel perchlorate suggests both that the boundary and fluid lipids have a different structural or dynamic organization and that they do not exchange effectively, at least on the $T_{1}$ timescale. This most probably is a result of peptide aggregation at the high GA/ DMPC ratio used here. In contrast, boundary lipids associated with integral transmembrane proteins, such as the myelin proteolipid protein, exchange with the bulk fluid lipids on a timescale comparable to that of the nitroxide spin-label $T_{1}$ relaxation [26,31].

\subsection{Spin-label binding to a protein: suppression of the unbound spin label}

Use of the out-of-phase $V_{1}^{\prime}$-mode allows one to select the EPR spectrum of the bound spin label, in the presence of free spin label in the aqueous phase. Fig. 8 shows the out-of-phase and in-phase first-harmonic EPR spectra of spin-labelled maleimide that is covalently bound to human serum albumin, but in the presence of excess unreacted spin label. The in-phase EPR spectrum (dotted line) consists of strongly immobilized and rapidly tumbling components that correspond to the bound (b) and the free $(f)$ species, respectively. Because the amplitude of the $V_{1}^{\prime}$-signal is strongly dependent on $T_{1}$, which is longer for the bound spin label, the relative amplitude of the immobilized component increases sub-

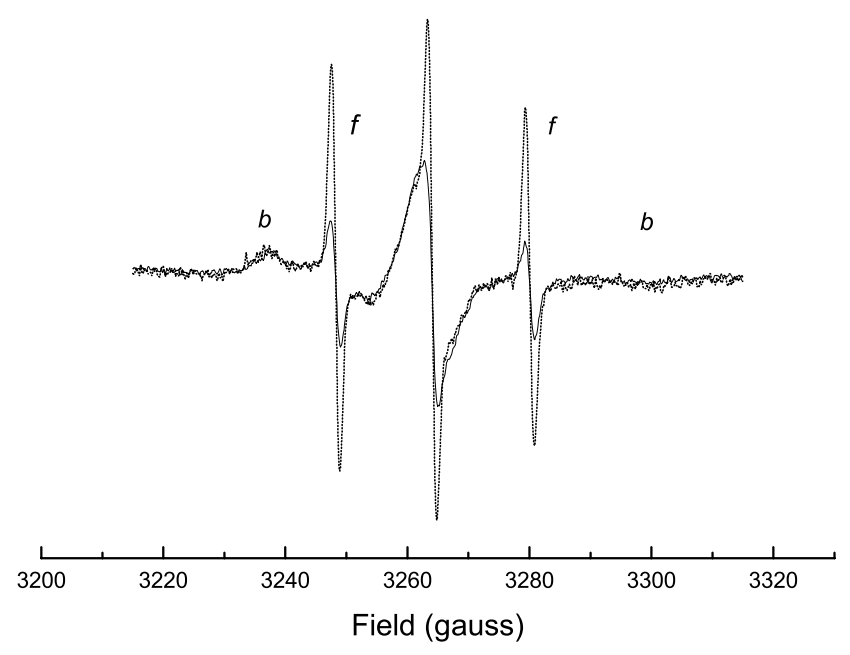

Fig. 8. $V_{1^{-}}$(dotted line) and $V_{1}^{\prime}$ - (solid line) EPR spectra of maleimide spin-labelled human serum albumin at $20^{\circ} \mathrm{C} . H_{1}=0.26 \mathrm{G} ; \omega_{m} /$ $2 \pi=100 \mathrm{kHz}$. Spectral components from the bound $(b)$ and free $(f)$ spin labels are indicated. stantially (by a factor 3-4x) as compared with that in the in-phase mode (see solid line in Fig. 8). The advantage of this approach compared with the exchange broadening of the conventional EPR spectra is that the non-linear method either does not require paramagnetic relaxant or that much smaller concentrations of relaxant are needed in order to quench the EPR signal of the free spin label.

\subsection{EPR spectra of spin labels in the presence of paramagnetic ions}

Our studies show that membranes containing paramagnetic ions with long spin-lattice relaxation times (viz., $\mathrm{Cu}^{2+}, \mathrm{Mn}^{2+}$, and $\mathrm{Gd}^{3+}$ ) give strong in-phase EPR signals in the same region as nitroxide spin labels [21]. In these cases, effective suppression of the signals from the paramagnetic ions can be achieved in the $V_{1}^{\prime}$-spectral mode. Spin-lattice and spin-spin relaxation times of these paramagnetic ions are still much shorter than those for nitroxide spin labels, and this results in a sharp decrease in relative amplitude of the background $V_{1}^{\prime}$-spectra from the paramagnetic ions.

Other important examples where this approach might be useful do not necessarily involve spin labels. In particular, it can be used for paramagnetic centers with different $T_{1}$ (e.g., non-heme iron centers in mitochondria, photosystem 1 of photosynthesis, etc.).

\section{Conclusions}

It is shown in this study that the first-harmonic outof-phase $V_{1}^{\prime}$-EPR signals can be a useful and valuable approach to detecting and separating overlapped EPR signals from species with different spin-lattice relaxation times. In general, the application of this approach is optimal at relatively low modulation frequencies and degrees of microwave saturation. However, in each case, tuning of these conditions is needed, at least when dealing with spin-label EPR spectra. In particular, an excessive reduction in $H_{1}$ or very short $T_{1}$ values of a spin label under study, may result in violation of the inequality: $\gamma_{e}^{2} H_{1}^{2} T_{1}^{2} \gg 1$, which would lead to changes in the $V_{1}^{\prime}$-lineshape. Use of selective paramagnetic relaxation agents, both extramembrane and intramembrane, serves further to enhance separation of signals in the $V_{1}^{\prime}$-EPR spectrum.

\section{Acknowledgments}

This study was supported in part by the Deutsche Forschungsgemeinschaft and the Russian Foundation for Basic Research, Grant 04-03-32631. 


\section{References}

[1] V.A. Livshits, T. Páli, D. Marsh, Spin relaxation measurements using first-harmonic out-of-phase absorption EPR signals, J. Magn. Reson. 134 (1998) 113-123.

[2] V.A. Livshits, D. Marsh, Spin relaxation measurements using first-harmonic out-of-phase absorption EPR signals: rotational motion effects, J. Magn. Reson. 145 (2000) 84-94.

[3] J.S. Hyde, D.D. Thomas, New EPR methods for the study of very slow motion: application to spin-labeled hemoglobin, Ann. N.Y. Acad. Sci. 222 (1973) 680-692.

[4] V.A. Livshits, D. Marsh, Fatty acid binding sites of serum albumin probed by non-linear spin-label EPR, Biochim. Biophys. Acta 1466 (2000) 350-360.

[5] V.A. Livshits, B.G. Dzikovski, D. Marsh, Anisotropic motion effects in CW non-linear EPR spectra: relaxation enhancement of lipid spin labels, J. Magn. Reson. 162 (2003) 429-442.

[6] B.G. Dzikovski, V.A. Livshits, D. Marsh, Oxygen permeation profile in lipid membranes: non-linear spin-label EPR, Biophys. J. 85 (2003) 1005-1012.

[7] V.A. Livshits, D. Marsh, Out-of-phase EPR spectra for measuring spin relaxation and separating the overlapped spin label EPR signals, in: Abstracts of the 33rd International Meeting and 4th Meeting of European Federation of EPR groups (EFEPR): Prospects for EPR spectroscopy in 21st century, Norwich (England) (2000) P35

[8] K. Halbach, Über eine neue Methode zur Messung von Relaxationszeiten und über den Spin von $\mathrm{Cr}^{53}$, Helv. Phys. Acta 27 (1954) 259-282.

[9] T. Páli, V.A. Livshits, D. Marsh, Dependence of saturationtransfer EPR intensities on spin-lattice relaxation, J. Magn. Reson. B 113 (1996) 151-159.

[10] D.D. Thomas, L.R. Dalton, J.S. Hyde, Rotational diffusion studied by passage saturation transfer electron paramagnetic resonance, J. Chem. Phys. 65 (1976) 3006-3024.

[11] J.S. Hyde, W. Froncisz, C. Mottley, Pulsed ELDOR measurement of nitrogen $T_{1}$ in spin labels, Chem. Phys. Lett. 110 (1984) 621-625.

[12] B.H. Robinson, D.A. Haas, C. Mailer, Molecular dynamics in liquids: spin-lattice relaxation of nitroxide spin labels, Science 263 (1994) 490-493.

[13] D. Marsh, T. Páli, L.I. Horváth, Progressive saturation and saturation transfer EPR for measuring exchange processes and proximity relations in membranes, in: L.J. Berliner (Ed.), Spin Labeling. The Next Millenium, Plenum Press, New York, 1998, pp. 23-82.

[14] T.G. Castner Jr., Saturation of the paramagnetic resonance of a V center, Phys. Rev. 115 (1959) 1506-1515.

[15] D. Marsh, A. Watts, Spin-labeling and lipid-protein interactions in membranes, in: P.C. Jost, O.H. Griffith (Eds.), Lipid-Protein Interactions, vol. 2, Wiley-Interscience, New York, 1982, pp. 53126.

[16] J.C. Bailar Jr., E.M. Jones, 13. Trioxalato salts (trioxalatoaluminiate, -ferriate, -chromiate, and -cobaltiate), Inorg. Synth. 1 (1939) $35-38$.
[17] P. Fajer, D. Marsh, Microwave and modulation field inhomogeneities and the effect of cavity $Q$ in saturation transfer ESR spectra. Dependence on sample size, J. Magn. Reson. 49 (1982) 212-224.

[18] W.L. Hubbell, C. Altenbach, Site-directed spin-labeling of membrane proteins, in: S.H. White (Ed.), Membrane Protein Structure: Experimental Approaches, Oxford University Press, New York, 1994, pp. 224-248.

[19] D. Marsh, Handbook of Lipid Bilayers, CRC Press, Boca Raton, FL, 1990.

[20] A.D. Keith, W. Snipes, Viscosity of cellular protoplasm, Science 183 (1974) 666-668.

[21] V.A. Livshits, B.G. Dzikovski, D. Marsh, Mechanism of relaxation enhancement of spin labels in membranes by paramagnetic ion salts: dependence on $3 d$ and $4 f$ ions and on the anions, J. Magn. Reson. 148 (2001) 221-237.

[22] M. Ge, J.H. Freed, An electron spin resonance study of interactions between gramicidin $\mathrm{A}$ and phosphatidylcholine bilayers, Biophys. J. 65 (1993) 2106-2123.

[23] M.T. Ge, J.H. Freed, Electron spin resonance study of aggregation of gramicidin in dipalmitoylphosphatidylcholine bilayers and hydrophobic mismatch, Biophys. J. 76 (1999) 264 280.

[24] A.J. Costa-Filho, R.H. Crepeau, P.P. Borbat, M. Ge, J.H. Freed, Lipid-gramicidin interactions: dynamic structure of the boundary lipid by 2D-ELDOR, Biophys. J. 84 (2003) 3364-3378.

[25] Z. Kóta, T. Páli, D. Marsh, Orientation and lipid-peptide interactions of gramicidin A in lipid membranes: polarized ATR infrared spectroscopy and spin-label electron spin resonance, Biophys. J. 86 (2004) 1521-1531.

[26] D. Marsh, L.I. Horváth, Structure, dynamics and composition of the lipid-protein interface. Perspectives from spin-labelling, Biochim. Biophys. Acta 1376 (1998) 267-296.

[27] D. Rice, E. Oldfield, Deuterium nuclear magnetic resonance studies of the interaction between dimyristoylphosphatidylcholine and gramicidin A, Biochemistry 18 (1979) 3272-3279.

[28] S. Rajan, S.Y. Kang, H.S. Gutowsky, E. Oldfield, Phosphorus nuclear magnetic resonance study of membrane structureinteractions of lipids with protein, polypeptide, and cholesterol, J. Biol. Chem. 256 (1981) 1160-1166.

[29] P.I. Watnick, S.I. Chan, P. Dea, Hydrophobic mismatch in gramicidin A/lecithin systems, Biochemistry 29 (1990) 62156221.

[30] M.R.R. de Planque, D.V. Greathouse, R.E. Koeppe II, H. Schäfer, D. Marsh, J.A. Killian, Influence of lipid/peptide hydrophobic mismatch on the thickness of diacylphosphatidylcholine bilayers. A ${ }^{2} \mathrm{H}$ NMR and ESR study using designed transmembrane $\alpha$-helical peptides and gramicidin A, Biochemistry 37 (1998) 9333-9345.

[31] L.I. Horváth, P.J. Brophy, D. Marsh, Exchange rates at the lipidprotein interface of the myelin proteolipid protein determined by saturation transfer electron spin resonance and continuous wave saturation studies, Biophys. J. 64 (1993) 622-631. 